Case Report

\title{
Successful Use of Hyperbaric Oxygen as Adjunctive Therapy for a Nonhealing Venous Ulcer in a Patient with Systemic Sclerosis and Pulmonary Arterial Hypertension: A Case Report and Review of the Literature
}

\author{
Isaac Biney (D, ${ }^{1}$ Tina Dudney, ${ }^{1}$ Mitchell Goldman, ${ }^{2}$ Lee Carder, ${ }^{2}$ and Elise Schriver ${ }^{1}$ \\ ${ }^{1}$ Department of Medicine, Division of Pulmonary Disease and Critical Care Medicine, University of Tennessee Graduate School \\ of Medicine, Knoxville, TN, USA \\ ${ }^{2}$ Department of Surgery, University of Tennessee Graduate School of Medicine, Knoxville, TN, USA
}

Correspondence should be addressed to Isaac Biney; isaacbiney@yahoo.co.uk

Received 3 December 2019; Accepted 7 February 2020; Published 24 February 2020

Academic Editor: Takeshi Terashima

Copyright () 2020 Isaac Biney et al. This is an open access article distributed under the Creative Commons Attribution License, which permits unrestricted use, distribution, and reproduction in any medium, provided the original work is properly cited.

\begin{abstract}
Skin ulcers are a common complication of systemic sclerosis (SSc) that can significantly impact the quality of life. There have been recent reports on the use of hyperbaric oxygen therapy $\left(\mathrm{HBO}_{2} \mathrm{~T}\right)$ in the management of nonhealing systemic sclerosis skin ulcers. The effect of $\mathrm{HBO}_{2} \mathrm{~T}$ on pulmonary arterial hypertension $(\mathrm{PAH})$, another common and potentially life-threatening complication of SSc, is unclear with literature on the subject lacking. We present the case of a 65-year-old female with limited SSc complicated by severe $\mathrm{PAH}$ and a nonhealing left lower extremity venous ulcer. $\mathrm{HBO}_{2} \mathrm{~T}$ was successfully used as an adjunct in her management resulting in complete resolution of the venous ulcer and improved quality of life without any adverse effects on her pulmonary arterial hypertension.
\end{abstract}

\section{Introduction}

Systemic sclerosis (SSc) is a chronic connective tissue disease with multiorgan involvement characterized by diffuse microangiopathy and the deposition of collagen into the skin and internal organs leading to progressive fibrosis. It is a subtype of a heterogeneous group of autoimmune diseases referred to as scleroderma and can be subcategorized by the degree of skin and internal organ fibrosis into limited SSc and diffuse SSc [1]. Skin ulcers are recorded in up to $50 \%$ of patients with SSc, and pathogenic mechanisms include microvascular damage and vasospasm [2]. Vascular impairment may also cause nonhealing of ulcers arising due to other mechanisms. These ulcers can be very painful and can cause a significant decline in a patient's quality of life. Management of these ulcers can be very challenging, and therapeutic guidelines remain controversial [2].

Hyperbaric oxygen therapy $\left(\mathrm{HBO}_{2} \mathrm{~T}\right)$ involves the administration of $100 \%$ oxygen in a closed chamber pressur- ized to greater than 1 atmosphere absolute (ATA). Its use in ischemic and nonhealing wounds has been documented since the mid- $20^{\text {th }}$ century, and there are well-established indications [3]. Its benefits for these indications have been attributed to antihypoxic, antimicrobial, antiedema, antiinflammatory, and angiogenetic effects [4]. Recently, there have been several case reports of successful use of $\mathrm{HBO}_{2} \mathrm{~T}$ in nonhealing ulcers, involving both the digits and extremities, in patients with SSc [4-6].

One relatively common and harmful complication of SSc is pulmonary arterial hypertension (PAH). Prevalence rates up to $19 \%$ have been reported in the literature and it is stated as the leading cause of death in this population [7-12]. There have been concerns raised by experts about the safety of $\mathrm{HBO}_{2} \mathrm{~T}$ in patients with significant $\mathrm{PAH}$. In a recent study looking at $\mathrm{HBO}_{2}$ T-treated ulcers in SSc subjects, patients with moderate to severe pulmonary hypertension were excluded [13]. There is currently no literature evaluating effects of $\mathrm{HBO}_{2} \mathrm{~T}$ in patients with $\mathrm{PAH}$. 
We report a case of successful use of $\mathrm{HBO}_{2} \mathrm{~T}$ as adjunctive therapy for the treatment of a nonhealing venous ulcer in a patient with limited SSc complicated by PAH.

\section{Case Presentation}

A 65-year-old female was diagnosed with limited systemic sclerosis 11 years previously after presenting with Raynaud's phenomenon complicated by digital necrosis resulting in amputation of the tip of her left third finger and interstitial lung disease. Her SSc had been managed with various disease-modifying agents, but each had to be discontinued due to intolerance. She was maintained on chronic lowdose prednisone. Her first encounter with our facility was when she presented to vascular surgery with chronic venous insufficiency and a nonhealing venous ulcer on the medial aspect of her left lower leg which had developed in October 2016. At the time, her functional status had declined from being fully functional to being confined to a wheelchair or a walker due to the discomfort from her leg ulcer and exertional dyspnea. She had debridement of the wound and radiofrequency ablation of her left saphenous vein and developed hypotension following the procedure requiring ICU admission. During workup for the hypotension, she had an echocardiogram which showed a left ventricular ejection fraction (LVEF) of 30-35\%, a severely dilated right ventricle (RV) with moderately reduced function as measured by a tricuspid annular plane systolic excursion (TAPSE) of $1.1 \mathrm{~cm}$ and an estimated right ventricular systolic pressure of $66 \mathrm{mmHg}$. A noncontrast CT scan of her chest showed mild interstitial lung disease. She subsequently had a right heart catheterization which showed a pulmonary artery pressure of $91 / 37 \mathrm{mmHg}$ with a mean of 55 , a transpulmonary gradient of $47 \mathrm{mmHg}$, a pulmonary capillary wedge pressure of $8 \mathrm{mmHg}$, and a pulmonary vascular resistance of 18.1 Woods units. Her cardiac output was $2.6 \mathrm{~L} / \mathrm{min}$ and cardiac index was $1.8 \mathrm{~L} / \mathrm{min} / \mathrm{m}^{2}$. She had a normal ventilation perfusion scan. She was diagnosed with PAH with a World Health Organization (WHO) functional class of IV. A repeat echocardiogram prior to the initiation $\mathrm{PAH}$ therapy showed a LVEF of 55-60\%. The patient refused parenteral prostanoids. She was treated with sildenafil and macitentan and inhaled treprostinil with improvement of her WHO functional class to class III.

She began treatment of her left lower extremity wound at our wound care center in April 2017. At her initial evaluation, her wound measured $10 \mathrm{~cm}$ long, $5.3 \mathrm{~cm}$ wide, and $0.3 \mathrm{~cm}$ deep with an area of $41.626 \mathrm{~cm}^{2}$ and a volume of $12.488 \mathrm{~cm}^{3}$. It was described as a full-thickness wound without exposed structures, with well-defined borders, a large amount of serosanguineous exudate and with medium amount of granulation tissue (Figure 1). Initial treatment was with nonadherent Silvercel as the primary dressing and Kerlix with ABD pad applied as the secondary dressing. After 2 months, the primary dressing was changed to the bioengineered skin substitute Apligraf and Mepitel One. Three months later, it was switched to Adaptic. The maximum reduction in wound area achieved with these modalities was $78.1 \%$ reduction after 7 months of treatment. The patient

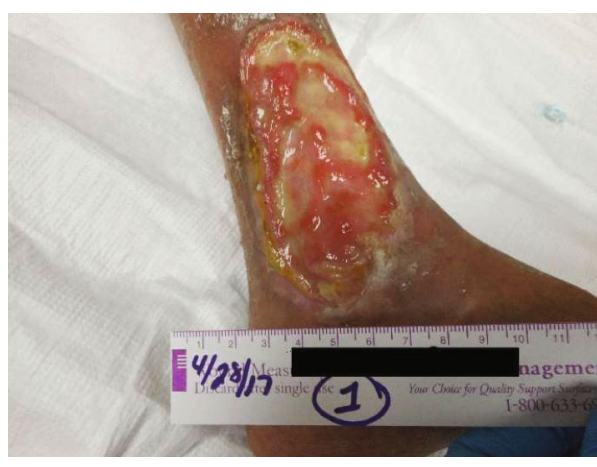

FIGURE 1: Left lower extremity venous ulcer at the time of initial presentation to the wound care center.

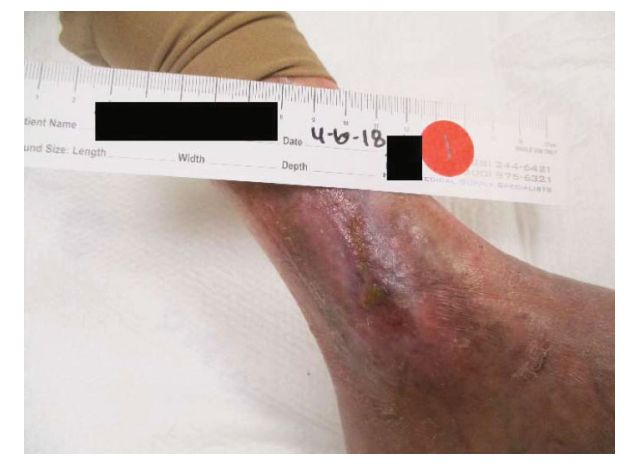

Figure 2: Left lower extremity demonstrating complete resolution of venous ulcer after a year of treatment.

was thought to have exhausted her options at this point. After a thorough search of the literature, consulting with the patient's pulmonologist and cardiologist, and detailed discussion with the patient outlining the potential risks involved and possible benefits, a joint decision was made to attempt $\mathrm{HBO}_{2} \mathrm{~T}$ which started in November 2017. Her most recent echocardiogram had been obtained 4 months prior, and it showed an LVEF of $61 \%$, grade 1 diastolic dysfunction, a moderately dilated RV with mildly reduced function, TAPSE $1.6 \mathrm{~cm}$, and an estimated RVSP of $72 \mathrm{mmHg}$. The patient received 30 treatments between 11/13/2017 and 12/19/2017 in a monoplace chamber at 2 ATA with 90 minutes of oxygen with no air breaks. Duration of each treatment ranged from 106 to 110 minutes. No immediate adverse events were noted during any of the treatment sessions, and she did not experience any worsening of her exercise tolerance. After completion of $\mathrm{HBO}_{2} \mathrm{~T}$, wound care continued with Apligraf, Adaptic, and Xeroform. Complete wound closure was achieved by April 2018 (Figure 2). She had 3 wound debridements during her treatment at the wound care center, 2 while receiving $\mathrm{HBO}_{2} \mathrm{~T}$. At follow-up, her functional status has improved to WHO class II, echocardiogram showed a LVEF of 65-70\%, mild left atrial enlargement, grade II diastolic dysfunction, mild right atrial enlargement, and a mildly to moderately dilated right ventricle with normal function measured by a TAPSE of $1.9 \mathrm{~cm}$. She had started exercising on a stationary bicycle and worked 5-7 hours per day. Prior to $\mathrm{HBO}_{2} \mathrm{~T}$, significant nocturnal wound pain 
interfered with her sleep. After wound closure, her pain resolved and quality of life vastly improved. The patient continues to do well. At her most recent follow-up in October 2019, her functional status remains at WHO class II, serum brain natriuretic peptide level was $45 \mathrm{pg} / \mathrm{mL}$, and her echocardiogram showed a normal-sized RV with normal function and an RVSP of $33.9 \mathrm{mmHg}$.

\section{Discussion}

Ulcers related to SSc (SSc-SU) have been known to be a challenging manifestation of the disease, often difficult to heal and recurrent. Their management has improved over the last decade with a multidisciplinary approach involving the combined use of systemic and advanced local treatments but has still been described as suboptimal [2]. Though our patient had a venous ulcer, similar challenges can be encountered in a patient with SSc as the disease has been associated with poor wound healing on account of the vasculopathy.

There has recently been developing interest in the use of $\mathrm{HBO}_{2} \mathrm{~T}$ in patients with SSc with intractable ulcers with several published case reports demonstrating its success. In our patient, $\mathrm{HBO}_{2} \mathrm{~T}$ was successfully used as an adjunct for treatment of her nonhealing ulcer [4-6]. Some experts have expressed concerns about the use of $\mathrm{HBO}_{2} \mathrm{~T}$ in patients with $\mathrm{PAH}$. Whether this is a generalized opinion or not, there have been no human studies evaluating this or reports of adverse events of $\mathrm{HBO}_{2} \mathrm{~T}$ when used in patients with $\mathrm{PAH}$. A recent study found that patients with SSc complicated by skin ulcers were more likely to have increased pulmonary arterial pressures [2]. Most studies looking at ulcers in systemic sclerosis have focused on digital ulcers with very little attention to nonhealing lower extremity ulcers [14]. Development of PAH and right ventricular dysfunction are likely to add to the challenge of managing such ulcers. In a study evaluating lower extremity ulcers in patients with SSc, 10 out of 249 patients had active nondigital leg ulcers. Of these, one patient had PAH and venous insufficiency and was among the 3 patients who had a nonhealing ulcer [14]. It is reasonable to deduce that patients with $\mathrm{PAH}$ will form a significant proportion of patients with SSc-SU who get to the point of being considered for $\mathrm{HBO}_{2} \mathrm{~T}$ if indeed $\mathrm{HBO}_{2} \mathrm{~T}$ becomes a standard adjunctive therapy in intractable SSc-SU.

There is scant literature evaluating the effect of $\mathrm{HBO}_{2} \mathrm{~T}$ on the pulmonary circulation in humans. Pulmonary oxygen toxicity manifesting as an acute exudative phase and subacute proliferative phase occurs with increasing fraction of inspired oxygen and partial pressure of oxygen. However, current applications of $\mathrm{HBO}_{2}$ T have not been shown to cause pulmonary symptoms or clinically significant pulmonary function defects [15]. Weaver et al. reported pulmonary artery catheter data in 10 normal subjects exposed to 40 minutes of hyperbaric air followed by 40 minutes of $\mathrm{HBO}_{2} \mathrm{~T}$. Pulmonary vascular resistance (PVR) decreased by up to $48 \%$ during $\mathrm{HBO}_{2} \mathrm{~T}$ with reduction in pulmonary artery pressure (PAP) by up to $19 \%$. Measurements returned to baseline after removal of hyperbaric conditions [16]. Long-term effects are unknown. In a study evaluating the effect of hyperoxia on the pulmonary circulation in rats, pulmonary vascular remodel- ing with altered hemodynamics including an increase in PVR and PAP was observed after continuous exposure to hyperoxia for 7 days at normobaric pressure [17]. Jacobson et al. demonstrated the development of pulmonary hypertension in rabbits after hyperbaric exposure to $100 \%$ oxygen for 1 hour with evidence to suggest that this was due to increased synthesis of thromboxane [18]. Armstrong et al. evaluated the safety of $\mathrm{HBO}_{2} \mathrm{~T}$ in SSc patients. Two patients were said to have not received $\mathrm{HBO}_{2} \mathrm{~T}$ on account of moderate to severe pulmonary hypertension, the underlying reason for this was not discussed. There have been case reports associating $\mathrm{HBO}_{2} \mathrm{~T}$ with pulmonary edema in patients with leftsided heart failure $[19,20]$. Possible mechanisms attributed to this finding include increased peripheral vasoconstriction leading to increased cardiac afterload, increased oxidative myocardial stress, decreased left ventricular (LV) compliance by oxygen radical-mediated reduction in nitric oxide right and left ventricular imbalance, and increased pulmonary capillary permeability. A study of the effect of $\mathrm{HBO}_{2} \mathrm{~T}$ on cardiac performance in anesthetized dogs showed increased systemic vascular resistance but no change in PVR, and the data suggested that $\mathrm{HBO}_{2}$ T may act by a differential effect on the autonomic innervation of the right and left ventricles [21].

Our successful use of $\mathrm{HBO}_{2} \mathrm{~T}$ as adjunctive therapy in our patient suggests that it may be safe to use in patients with $\mathrm{PAH}$, but further research is needed to fully evaluate this as the risk benefit is unknown. With increasing interest in $\mathrm{HBO}_{2} \mathrm{~T}$ use in SSc-SU and with the high prevalence of $\mathrm{PAH}$ in this population, it will be important to not automatically exclude patients who could benefit from a potentially life-changing therapy.

\section{Conflicts of Interest}

The authors declare that they have no conflicts of interest.

\section{References}

[1] C. Ferreli, G. Gasparini, A. Parodi, E. Cozzani, F. Rongioletti, and L. Atzori, "Cutaneous manifestations of scleroderma and scleroderma-like disorders: a comprehensive review," Clinical Reviews in Allergy \& Immunology, vol. 53, no. 3, pp. 306336, 2017.

[2] D. Giuggioli, A. Manfredi, F. Lumetti, M. Colaci, and C. Ferri, "Scleroderma skin ulcers definition, classification and treatment strategies our experience and review of the literature," Autoimmunity Reviews, vol. 17, no. 2, pp. 155-164, 2018.

[3] G. Lam, R. Fontaine, F. L. Ross, and E. S. Chiu, "Hyperbaric oxygen therapy: exploring the clinical evidence," Advances in Skin \& Wound Care, vol. 30, no. 4, pp. 181-190, 2017.

[4] B. Mirasoglu, B. S. Bagli, and S. Aktas, "Hyperbaric oxygen therapy for chronic ulcers in systemic sclerosis - case series," International Journal of Dermatology, vol. 56, no. 6, pp. 636640, 2017.

[5] Y. M. Markus, M. J. Bell, and A. W. Evans, "Ischemic scleroderma wounds successfully treated with hyperbaric oxygen therapy," The Journal of Rheumatology, vol. 33, no. 8, pp. 1694-1696, 2006. 
[6] E. Poirier, H. Wind, and N. Cordel, "Efficacy of hyperbaric oxygen therapy in the treatment of ischemic toe ulcer in a patient presenting systemic sclerosis," Annales de dermatologie et de venereologie, vol. 144, no. 1, pp. 55-59, 2017.

[7] G. Lefèvre, L. Dauchet, E. Hachulla et al., "Survival and prognostic factors in systemic sclerosis-associated pulmonary hypertension: a systematic review and meta-analysis," Arthritis and Rheumatism, vol. 65, no. 9, pp. 2412-2423, 2013.

[8] V. D. Steen and T. A. Medsger, "Changes in causes of death in systemic sclerosis, 1972-2002," Annals of the Rheumatic Diseases, vol. 66, no. 7, pp. 940-944, 2007.

[9] J. G. Coghlan, C. P. Denton, E. Grünig et al., "Evidence-based detection of pulmonary arterial hypertension in systemic sclerosis: the DETECT study," Annals of the Rheumatic Diseases, vol. 73, no. 7, pp. 1340-1349, 2014.

[10] C. Muangchan, Canadian Scleroderma Research Group, M. Baron, and J. Pope, "The 15\% rule in scleroderma: the frequency of severe organ complications in systemic sclerosis. A systematic review," The Journal of Rheumatology, vol. 40, no. 9, pp. 1545-1556, 2013.

[11] L. Schachna, F. M. Wigley, B. Chang, B. White, R. A. Wise, and A. C. Gelber, "Age and risk of pulmonary arterial hypertension in scleroderma," Chest, vol. 124, no. 6, pp. 2098-2104, 2003.

[12] K. Morrisroe, the Australian Scleroderma Interest Group (ASIG), M. Huq et al., "Risk factors for development of pulmonary arterial hypertension in Australian systemic sclerosis patients: results from a large multicenter cohort study," $B M C$ Pulmonary Medicine, vol. 16, no. 1, p. 134, 2016.

[13] S. Armstrong, A. W. Evans, Z. Ahmad, and S. R. Johnson, "Safety and effectiveness of hyperbaric oxygen therapy for systemic sclerosis ulcers," 2016, https://acrabstracts.org/abstract/ safety-and-effectiveness-of-hyperbaric-oxygen-therapy-forsystemic-sclerosis-ulcers/.

[14] V. K. Shanmugam, P. Price, C. E. Attinger, and V. D. Steen, "Lower extremity ulcers in systemic sclerosis: features and response to therapy," International Journal of Rheumatology, vol. 2010, Article ID 747946, 8 pages, 2010.

[15] M. Heyboer III, D. Sharma, W. Santiago, and N. McCulloch, "Hyperbaric oxygen therapy: side effects defined and quantified," Advances in Wound Care, vol. 6, no. 6, pp. 210-224, 2017.

[16] L. K. Weaver, S. Howe, G. L. Snow, and K. Deru, "Arterial and pulmonary arterial hemodynamics and oxygen delivery/extraction in normal humans exposed to hyperbaric air and oxygen," Journal of Applied Physiology, vol. 107, no. 1, pp. 336345, 2009.

[17] R. Jones, W. M. Zapol, and L. Reid, "Pulmonary artery remodeling and pulmonary hypertension after exposure to hyperoxia for 7 days. A morphometric and hemodynamic study," The American Journal of Pathology, vol. 117, no. 2, pp. 273-285, 1984.

[18] J. M. Jacobson, J. R. Michael, R. A. Meyers, M. B. Bradley, A. M. Sciuto, and G. H. Gurtner, "Hyperbaric oxygen toxicity: role of thromboxane," Journal of Applied Physiology, vol. 72, no. 2, pp. 416-422, 1992.

[19] L. K. Weaver and S. Churchill, "Pulmonary edema associated with hyperbaric oxygen therapy," Chest, vol. 120, no. 4, pp. 1407-1409, 2001.
[20] C. Obiagwu, V. Paul, S. Chadha, G. Hollander, and J. Shani, "Acute pulmonary edema secondary to hyperbaric oxygen therapy," Oxford Medical Case Reports, vol. 2015, no. 2, pp. 183-184, 2015.

[21] F. L. Abel, J. E. McNamee, D. L. Cone, D. Clarke, and J. Tao, "Effects of hyperbaric oxygen on ventricular performance, pulmonary blood volume, and systemic and pulmonary vascular resistance," Undersea \& Hyperbaric Medicine, vol. 27, no. 2, pp. $67-73,2000$. 